\title{
Titanium Doped ITO Thin Films Produced by Sputtering Method
}

\author{
Leandro Voisin ${ }^{1}$, Makoto Ohtsuka ${ }^{2, *}$ and Takashi Nakamura ${ }^{2}$ \\ ${ }^{1}$ New Industry Creation Hatchery Center, Tohoku University, Sendai 980-8579, Japan \\ ${ }^{2}$ Institute of Multidisciplinary Research for Advanced Materials, Tohoku University, Sendai 980-8577, Japan
}

Indium-tin-oxide (ITO) thin films, typically produced by $\mathrm{In}_{2} \mathrm{O}_{3}-10$ mass $\% \mathrm{SnO}_{2}$ target, are widely used for the elaboration of optoelectronics devices such as liquid crystal displays (LCD), flat panel displays (FPD), plasma displays, touch panels, etc., and they are required to satisfied specific optoelectronical properties such as, low volume resistivity and high transmittance.

Due to the current high cost and limited supply of indium, titanium was investigated as dopant element for the production of ITO thin films. Two sputtering method were carried out; a combinatorial method where an $\mathrm{In}_{2} \mathrm{O}_{3}-10$ mass $\% \mathrm{SnO}_{2}$ and a Ti targets were simultaneously sputtered and then a co-sputtering method where, in addition to the previous targets, an $\mathrm{In}_{2} \mathrm{O}_{3}-50$ mass $\% \mathrm{SnO}_{2}$ and a TiO $\mathrm{O}_{2}$ targets were also sputtered.

Effects of the oxygen flow and heat treatment temperature on the optoelectronical properties of ITO thin films doped with Ti, as metal and dioxide, were investigated and the results were discussed on the basis of crystallization for bixbyte structure of $\operatorname{In}_{2} \mathrm{O}_{3}$. The obtained results indicate that the use of Ti as dopant element during the production of ITO thin films by sputtering method will be promising. [doi:10.2320/matertrans.MBW200907]

(Received October 15, 2009; Accepted December 7, 2009; Published January 27, 2010)

Keywords: titanium doped indium-tin-oxide thin film, combinatorial sputtering method, volume resistivity, transmittance

\section{Introduction}

Indium-tin-oxide (ITO) thin films are widely used as transparent conductive coating during the elaboration of optoelectronics devices such as liquid crystal displays (LCD), flat panel displays (FPD) ${ }^{1)}$ plasma displays, touch panels, electronic ink applications, solar cells, ${ }^{2)}$ antistatic coating and organic light emitting devices (OLED). ${ }^{3)}$ The typical ITO target used for the sputtering method presents a composition of $\mathrm{In}_{2} \mathrm{O}_{3}-10$ mass $\% \mathrm{SnO}_{2}$ and their produced thin films are required to satisfied specific values for several properties such as high electrical conductivity, $\sigma$, $(\sim 1000 \mathrm{mS})$, high optical transmittance, $\tau$, in the visible region of the spectrum $(>85 \%, 400 \sim 800 \mathrm{~nm})$, satisfactory etching quality, etc. ${ }^{4)}$

Since in recent times ITO has expected an increasing demand with the popularity of LCD computer monitors and televisions, the indium world supply is becoming scarce causing an important rise in its price. The amount of indium consumed is largely a function of worldwide ITO thin films production. Worldwide production of indium is currently 480 and 650 tons per year from mining and recycling, respectively. ${ }^{5)}$

The recent changes in demand and supply have resulted in high and fluctuating prices of indium, which from 2002 to 2007 ranged from US\$95/ $\mathrm{kg}$ to US $\$ 1000 / \mathrm{kg}$ with a current price around US\$510/.kg. ${ }^{6)}$ Demand for indium is expected to increase back with the actual increasing demand for ITO thin films for LCD production and with large-scale manufacture of copper indium gallium (di)selenide (CIGS)-based thin film solar technology starting by several companies since 2008 .

Based on content of indium in zinc ore stocks, there is a worldwide reserve base of approximately 6000 tons of economically-viable indium. ${ }^{7)}$ This value has led to estimates suggesting that, at current consumption rates, there is only 13 years supply of indium left, ${ }^{8)}$ which certainly represents a

*Corresponding author, E-mail: ohtsuka@tagen.tohoku.ac.jp strong negative environmental impact because its premature depletion. Therefore, in order to decrease the use of indium during the production of ITO films, a new target, which considers a smaller quantity of $\operatorname{In}_{2} \mathrm{O}_{3}$ in its composition and that it maintains or improved the related properties is required to be developed.

Titanium is a transition metal, which could be a suitable substitution or dopant element for the production of ITO thin films due to its quite low electrical volume resistivity, $\rho_{\mathrm{V}}$, $0.42 \mu \Omega \mathrm{m}$, relatively high transmittance and the excellent resistance to corrosion for its (IV) oxide. ${ }^{9)}$ In addition, titanium is the ninth-most abundant element in the Earth's crust $(0.63 \text { mass } \%)^{9)}$ and its actual commercial price is about US $\$ 19 / \mathrm{kg}^{6}$ ) 25 times cheaper than that of indium.

In the present research, the production of titanium-doped ITO thin films by using the sputtering method was investigated for developing a new target, which considers a lower content of indium. The effect of the heat treatment on the sputtered films was also investigated.

\section{Experimental Method and Procedure}

A schematic diagram of the sputtering apparatus (ULVAC, CS-200) used in the present study is shown in Fig. 1. Thin films were deposited on glass substrates (Corning EAGLE 2000, surface: $50 \mathrm{~mm} \times 50 \mathrm{~mm}$, thickness: $0.7 \mathrm{~mm}$ ). Two sputtering method were carried out; a combinatorial method where an $\mathrm{In}_{2} \mathrm{O}_{3}-10$ mass $\% \mathrm{SnO}_{2}$ (ITO [90]) and a Ti targets were simultaneously sputtered and then a co-sputtering method where, in addition to the previous targets, an $\mathrm{In}_{2} \mathrm{O}_{3}-50$ mass $\% \mathrm{SnO}_{2}$ (ITO [50]) and a $\mathrm{TiO}_{2}$ targets were also later sputtered. A brief description and the corresponding sputtering condition for each method are explained in sections 2.1 and 2.2 while their schematic diagrams are shown in Figs. 2 (a) and (b), respectively.

To sputter the ITO targets a resonance circuit $(\mathrm{L} / \mathrm{C})$ filter to eliminate ripple frequencies, an active arc killer (A2K) to prevent abnormal discharge, and a direct current (DC) power 


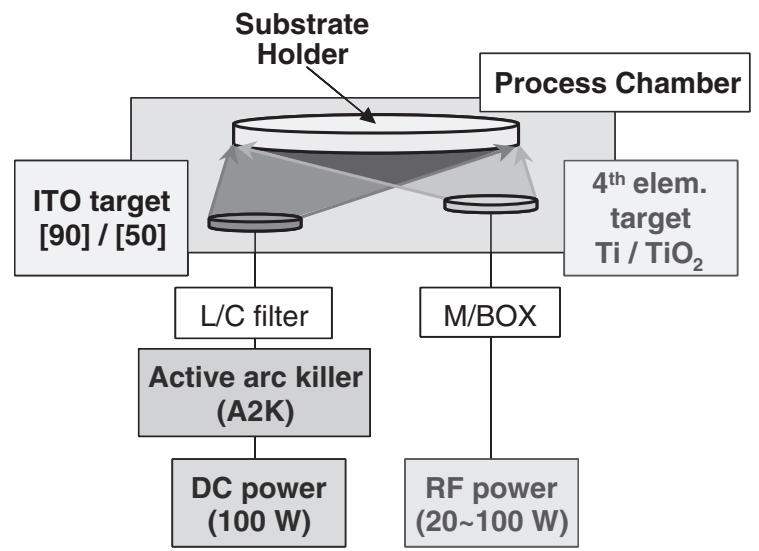

Fig. 1 Schematic diagram of the sputtering apparatus.

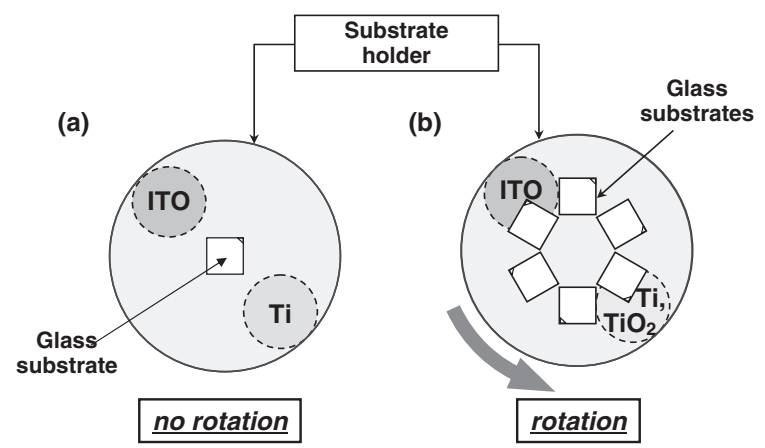

Fig. 2 Schematic diagram of sputtering methods. (a) Combinatorial and (b) co-sputtering.

source were available, while for Ti targets a matching box $(\mathrm{M} / \mathrm{BOX})$ and a high radio frequency $(\mathrm{RF})$ power source were used. Process chamber was vacuum at $10^{-5} \mathrm{~Pa}$ for its base pressure while total experimental pressures were resulted to be between 0.67 and $0.7 \mathrm{~Pa}$.

The effects of the oxygen flow, $Q\left(\mathrm{O}_{2}\right)$, and heat treatment temperature, $T_{\mathrm{HT}}$, on the optoelectronical properties of $\tau$ and $\rho_{\mathrm{V}}$ for the ITO thin films doped with Ti, as metal and dioxide, were investigated and compared against those previously obtained by the authors ${ }^{10)}$ for the ITO [90] thin film produced under the gas flow ratio condition of argon to oxygen, $\left(Q(\mathrm{Ar}) / Q\left(\mathrm{O}_{2}\right)\right),=50 / 0.2$, which resulted to be the best condition for that material.

The measurements of $\rho_{\mathrm{V}}$ were carried out with a resistivity meter (Mitsubishi chemical analytech, Loresta GP Model MCP-T610) by using a 4-terminal method, while $\tau$ were measured into the $200 \sim 900 \mathrm{~nm}$ range of wavelength $(\lambda)$ by using a Spectrophotometer (Hitachi High-Tech, U-3900H). For the latter, a glass substrate resulted to reach around $92 \%$ in the range of wavelength, $\lambda$, between 350 and $1000 \mathrm{~nm}$, this measurement and those for the obtained thin films were taken by consider air as reference.

\subsection{Combinatorial sputtering method}

By using the combinatorial sputtering method a composition gradient Ti doped ITO thin film can be produced on a substrate glass since both ITO [90] and high purity metallic Ti targets are sputtered at the same time keeping fixed the substrate holder during the deposition (Fig. 2 (a)).

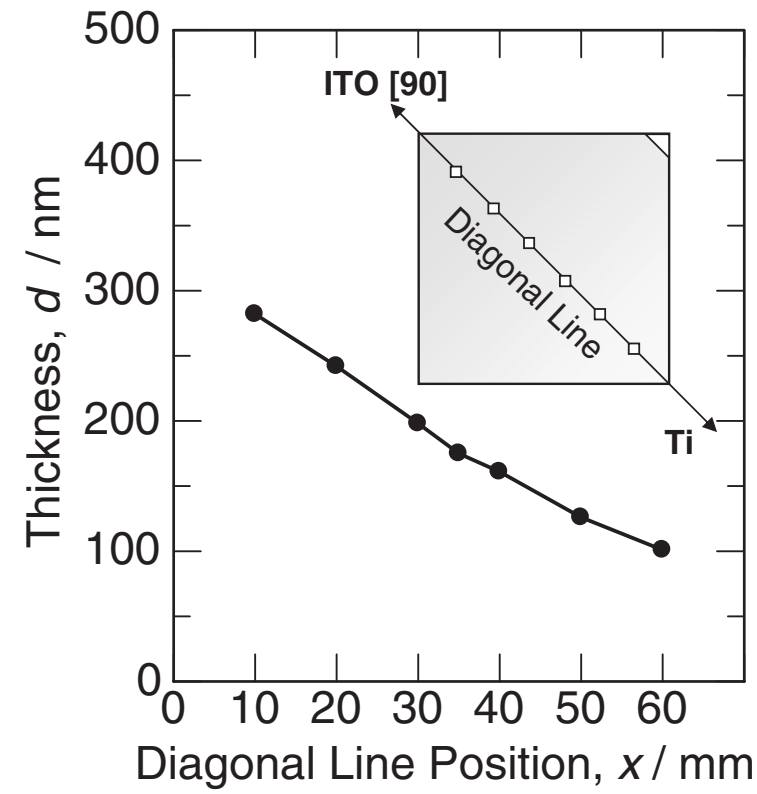

Fig. 3 Relation between $d$ and $x$ for Ti doped ITO [90] thin film produced by combinatorial sputtering method.

For combinatorial sputtered experiments, the substrate glass was located on the center of the substrate holder, at the point equidistant from ITO [90] and Ti targets, $\left(Q(\mathrm{Ar}) / Q\left(\mathrm{O}_{2}\right)\right)$ was set at 50/0.2 and sputtering time at $1.8 \mathrm{ks}$.

For the production of the ITO [90] film, the DC power source was fixed at $100 \mathrm{~W}$ and when the combinatorial sputtering method was applied in addition to the latter, the RF power source was set at $50 \mathrm{~W}$ for Ti.

In order to determine the gradient of thickness, $d$, for the obtained films, a very thin line of about $50 \mu \mathrm{m}$ of width and $1 \mathrm{~mm}$ of length was removed with a laser in six aligned positions, $x$, separated $10 \mathrm{~mm}$ to each other and located along the diagonal line between the targets and then $d$, was measured in each of those positions by using a scanning probe microscope (SPM, SII L-trace II), under dynamic force mode (DFM). The relation between $d$ and $x$ for the Ti doped ITO [90] film is shown in Fig. 3.

Finally, the produced films were heat treated at $523 \mathrm{~K}$ for $3.6 \mathrm{ks}$ (HT523) and by using the obtained thickness information, their composition and optoelectronical properties were determined for both as deposited, as-depo., and heat treated, HT, conditions.

\subsection{Co-sputtering method}

The co-sputtering method corresponds to simultaneously sputter the two target cathodes under the rotation of the substrate holder in order to obtain a homogeneous deposition of mixed composition (Fig. 2(b)). In this research, two cases were considered; the simultaneous sputtering of the ITO [90] with the pure metallic Ti targets, and that of the ITO [50] with the $\mathrm{TiO}_{2}$ targets. Rotation speed for the substrate holder was set to $40 \mathrm{rpm}$.

Deposition rates were determined by sputtering each single target at two different powers for $1.8 \mathrm{ks}$ and later measuring the thickness of the obtained films by using a SPM as it was previously explained in Section 2.1. In order to produce 
films with a standard thickness of about $150 \mathrm{~nm}$ and a mass $\%$ of $\mathrm{In}_{2} \mathrm{O}_{3}$ close to 48 , DC power was fixed to $100 \mathrm{~W}$ for ITO targets, RF power was fixed to 100 and $20 \mathrm{~W}$ for $\mathrm{Ti}$ and $\mathrm{TiO}_{2}$ target while sputtering time was set to 20 and $28 \mathrm{~min}$ for ITO [90]-Ti and for ITO [50]- $\mathrm{TiO}_{2}$ co-sputtered experiments, respectively.

To determine the effect of $Q\left(\mathrm{O}_{2}\right)$ on $\rho_{\mathrm{V}}$, three different levels for $Q\left(\mathrm{O}_{2}\right)$ of $0.2,0.3$ and 0.4 , and $0,0.2$ and $0.3 \mathrm{sccm}$ were investigated for ITO [90]-Ti and ITO [50]- $\mathrm{TiO}_{2}$ co-sputtered experiments, respectively. $Q(\mathrm{Ar})$ was fixed to $50 \mathrm{sccm}$ for all the experiments.

As is shown in Fig. 2(b), six glasses were concentrically set on the substrate holder, one film was kept for as-depo. condition, while the other five ones were later heat treated, HT, by separated at 523, 623, 723, 823 and $923 \mathrm{~K}$, respectively to investigate the effect of the $T_{\mathrm{HT}}$, on the optoelectronical properties of ITO thin films doped with $\mathrm{Ti}$, as metal and dioxide.

\section{Results and Discussions}

The effects of $Q\left(\mathrm{O}_{2}\right)$ and $T_{\mathrm{HT}}$ on the optoelectronical properties of $\sigma, \rho_{\mathrm{V}}$ and $\tau$ for the ITO thin films doped with Ti, as metal and dioxide, were investigated and the results were compared to those previously obtained by the authors ${ }^{10)}$ for the ITO [90] thin film and discussed on the basis of crystallization for bixbyte structure of $\mathrm{In}_{2} \mathrm{O}_{3}$.

\subsection{Ti doped ITO thin films produced by combinatorial method}

$\sigma$ was measured in six aligned positions, $x$, on the films separated $10 \mathrm{~mm}$ each other and located along the diagonal line between the targets. The results of $\sigma$, for the ITO [90] and Ti doped ITO [90] as-depo. and HT523 films are shown together in relation to $x$ in Fig. 4. With increasing the distance from the ITO [90] target, $\sigma$ decreases from 50 to $3.7 \mathrm{mS}$ and from 21 to $0.03 \mathrm{mS}$ in the ITO [90] and, Ti doped ITO [90] as-depo. films, respectively. It is considered that this difference in the latter may be ascribed to the stronger chemical affinity of oxygen for titanium than that for indium, thus the ITO [90] as-depo. film is reduced by the titanium resulting in the formation of the lower conductive $\mathrm{InO}$.

When the heat treatment was applied to the ITO [90] film, $\sigma$ resulted to be slightly decreased in the region close to the ITO [90] target but it was half order improved in the far away part. On the other hand, on the Ti-doped ITO [90] film, it causes an important improvement of $\sigma$ in the whole region, reaching even two orders of difference in the region close to the Ti target, this effect may be ascribed to the decrease of black indium monoxide ( $\mathrm{InO})$ in the film because the oxygen vacancy becomes filled under air atmosphere.

$\tau$ was measured for the ITO [90] and Ti doped ITO [90] as-depo. and HT523 films on the their lower-right part through a square area which was indicated with dashed line in Fig. 5. According to those results, $\tau$ is about 80 and $85 \%$ in the visible region of the spectrum $(400 \sim 800 \mathrm{~nm})$, for the ITO [90] and Ti-doped ITO [90] as-depo. films, and when the heat treatment was considered $\tau$ was improved to about 90 and $88 \%$, respectively. All the results are quite similar in tendency with the exception of that obtained for the ITO [90]

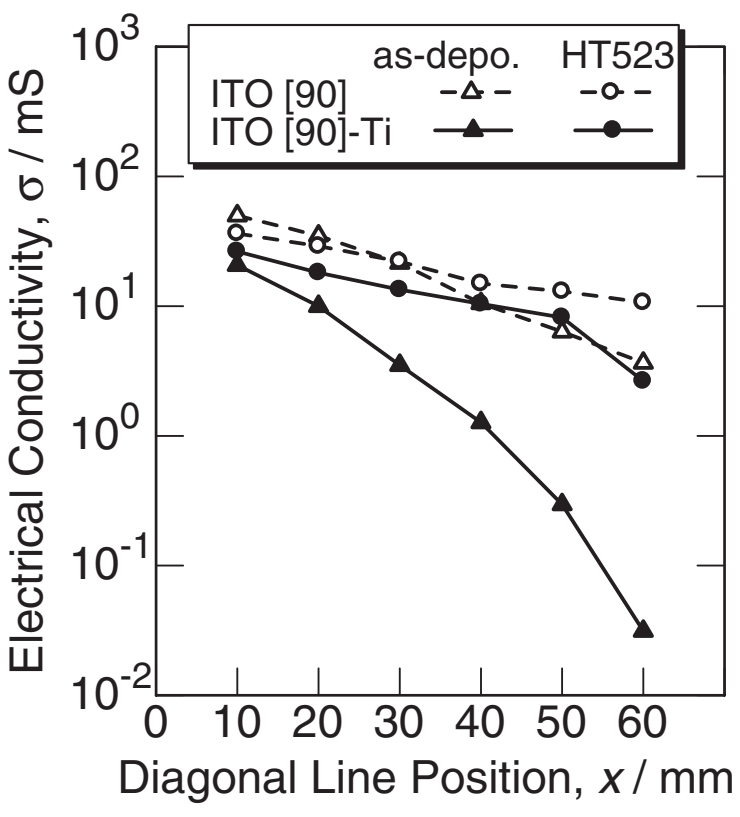

Fig. 4 Relation between $\sigma$ and $x$ for ITO [90] and Ti doped ITO [90] thin films produced by combinatorial sputtering method.

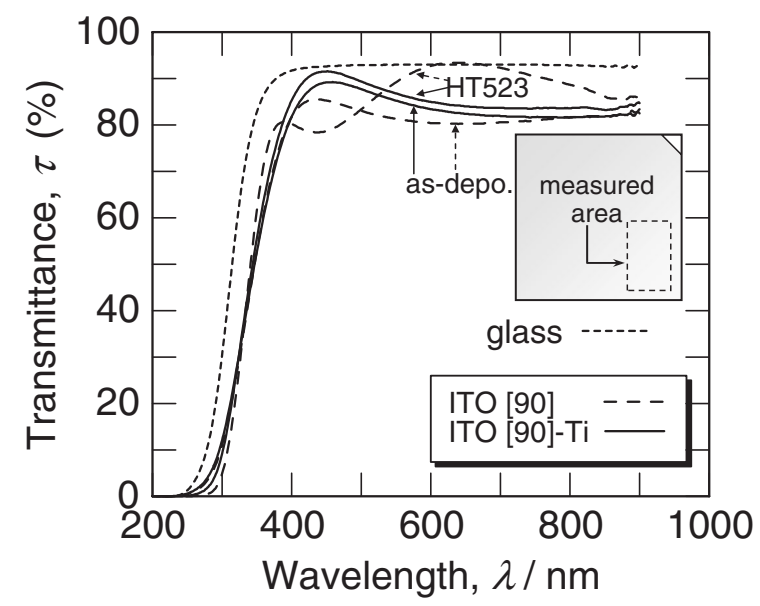

Fig. 5 Relation between $\tau$ and $\lambda$ for ITO [90] and Ti doped ITO [90] thin films produced by combinatorial sputtering method.

HT523 film, which presents a notorious imperfection in the range of $\lambda$ between 400 and $600 \mathrm{~nm}$.

Figure 6 shows the X-ray diffraction (XRD) results measured on the lower-right part of the films obtained by combinatorial sputtering method. According to Fig. 6, as-depo. films result in amorphous structure, nevertheless when heat treatment was applied to the films, five peaks appeared, which can be assigned to the cubic bixbyte structure of $\mathrm{In}_{2} \mathrm{O}_{3} .{ }^{11)}$ Non $\mathrm{SnO}_{2}$ peaks appeared and the intensity of the peaks for the HT films was higher in the Ti doped ITO [90] HT523 substrate.

Finally, according to Figs. 4 and 6, it can be observed that there is a clear relationship between $\sigma$ and crystallization for bixbyte, by heat treated the films, the concentration of dopants, tin or oxygen vacancy trapped at crystalline defects can be decreased, improving bixbyte crystallization and increasing electrically active species inside each grain. 


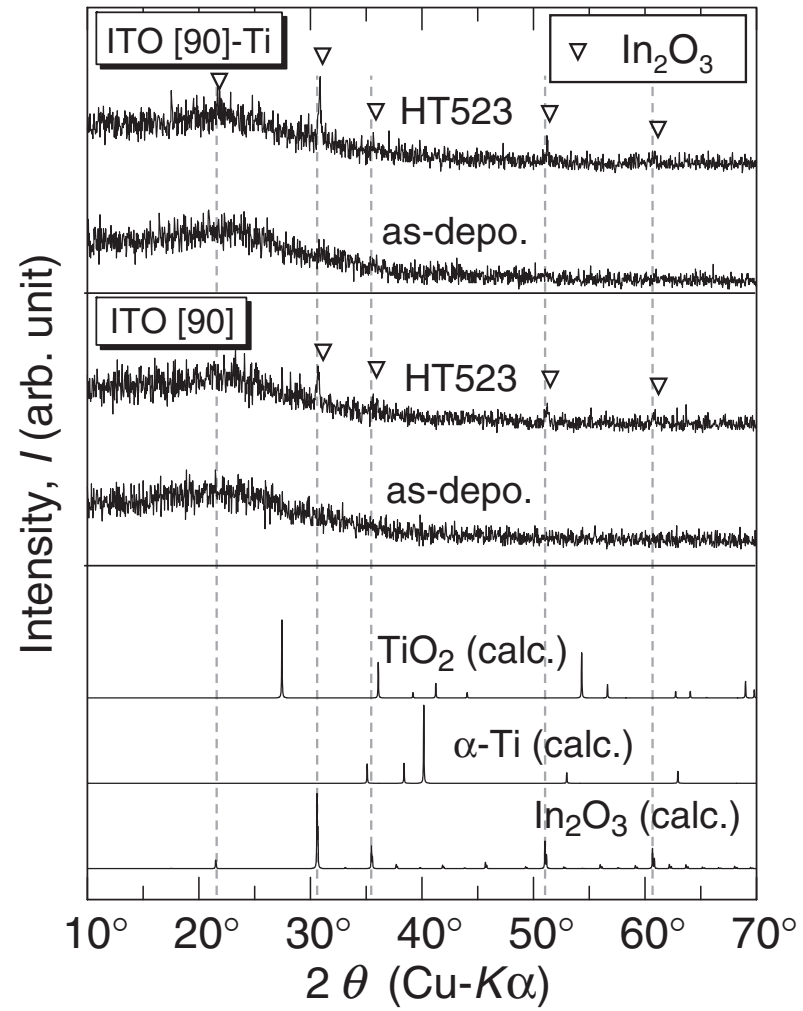

Fig. 6 XRD results for ITO [90] and Ti doped ITO [90] thin films produced by combinatorial sputtering method.

\subsection{Ti doped ITO thin films produced by co-sputtering method}

When the thickness was measured for each co-sputtered film, a barely small difference with a standard deviation between 0.23 and 0.57 depends on the sample was found, thus it was decided to measure three symmetric and equidistant points on both the left and the right sides of the films, six points in total, and take the average value as the corresponding thickness for each specific film. By using the thickness information, $\rho_{\mathrm{V}}$ was later determined for each obtained film.

Figure 7 shows the effects of $Q\left(\mathrm{O}_{2}\right)$ and $T_{\mathrm{HT}}$ on the $\rho_{\mathrm{V}}$ measured for the Ti doped ITO [90] films. According to Fig. 7, with increasing $Q\left(\mathrm{O}_{2}\right)$, in the system $\rho_{\mathrm{V}}$ strongly increased for the as-depo. film while for the HT films, $\rho_{\mathrm{V}}$ present quite similar tendencies each other showing a minimum at $Q\left(\mathrm{O}_{2}\right)=0.3 \mathrm{sccm}$. It was also observed that $\rho_{\mathrm{V}}$ decreases with increasing $T_{\mathrm{HT}}$, this effect may be ascribed to the improvement of $\operatorname{In}_{2} \mathrm{O}_{3}$ bixbyte crystallinity. The minimum value for $\rho_{\mathrm{V}}$ of $26.9 \mu \Omega \mathrm{m}(\sigma=25 \mathrm{mS})$ was obtained from the Ti doped ITO [90] HT923 film. This value is about twice, but still a promising result in comparison to that of $\rho_{\mathrm{V}}=14.3 \mu \Omega \mathrm{m}(\sigma=48 \mathrm{mS})$ obtained from the ITO [90] HT523 film and showed in the same figure with opened triangle mark.

$\tau$ was measured for the as-depo. and HT523 ITO [90] and Ti doped ITO [90] films under the condition of $Q(\mathrm{Ar}) /$ $Q\left(\mathrm{O}_{2}\right)=50 / 0.2$ and 50/0.3, respectively on the their upperleft part through a square area indicated with dashed line in Fig. 8. According to those results, Ti doped ITO [90] asdepo. film shows a $\tau$ with a concave form and values between

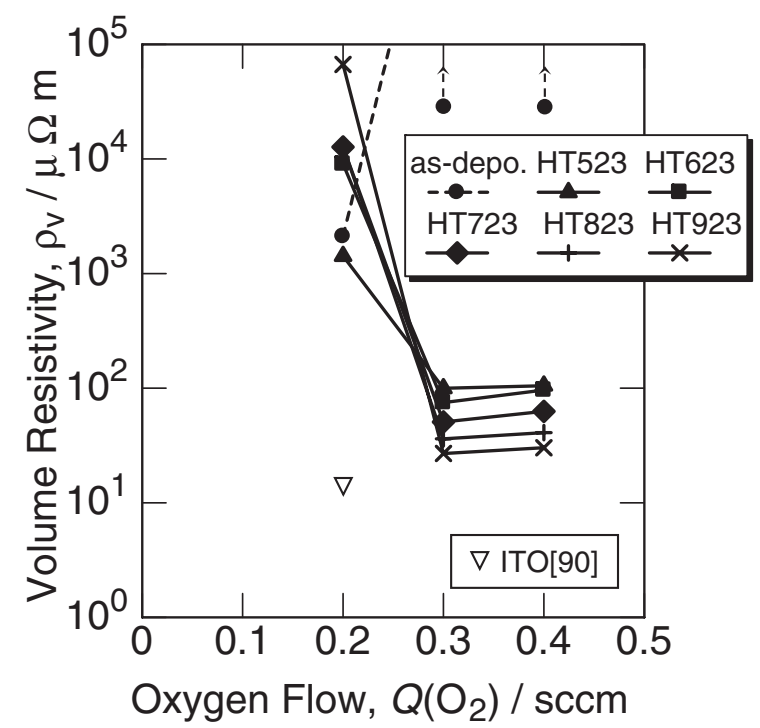

Fig. 7 Effects of $Q\left(\mathrm{O}_{2}\right)$ and $T_{\mathrm{HT}}$ on the $\rho_{\mathrm{V}}$ of Ti doped ITO [90] thin films produced by co-sputtering method.

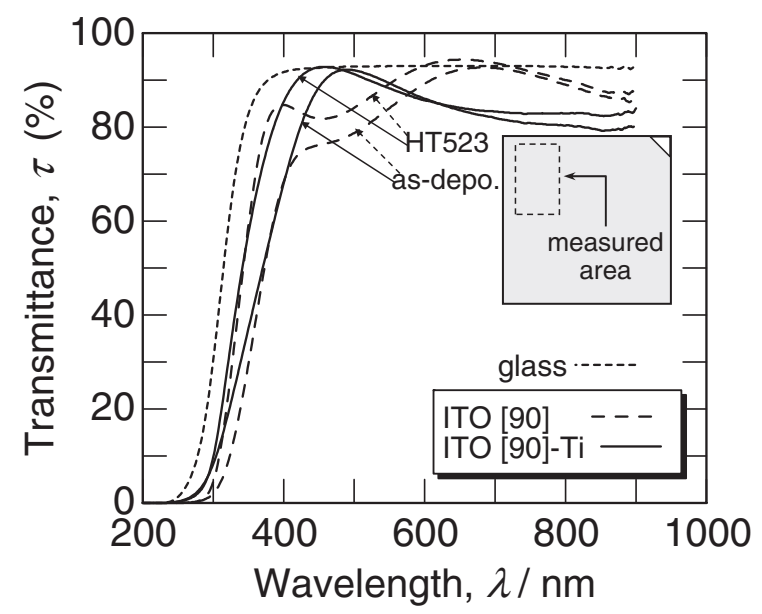

Fig. 8 Effects of $T_{\mathrm{HT}}$ on the $\tau$ of ITO [90] and Ti doped ITO [90] thin films produced by co-sputtering method, when $Q(\mathrm{Ar}) / Q\left(\mathrm{O}_{2}\right)=50 / 0.2$ and $50 / 0.3$, respectively.

85 and $90 \%$ in the $\lambda$ range between $400 \sim 550 \mathrm{~nm}$ reaching later a plateau until the $800 \mathrm{~nm}$. $\tau$ was not affected by temperature in the range between 523 and $923 \mathrm{~K}$ and so HT films show an almost identical $\tau$ each other and similar in tendency to that for as-depo. film but increasing the width of the concavity starting from $\lambda=350 \mathrm{~nm}$. In comparison to ITO [90], Ti doped ITO [90] films present a quite improved $\tau$ in the range of $\lambda$ between $400 \sim 550 \mathrm{~nm}$ and a similar tendency but lower of around $8 \%$ between $550 \sim 900 \mathrm{~nm}$. The improvement in the first range may be ascribed the higher $\tau$ of titanium dioxide than that of tin dioxide. It is important to note that the $\tau$ for the HT523 ITO [90] film resulted to be higher than that of the glass substrate in the range of $\lambda$ between $600 \sim 700 \mathrm{~nm}$, this effect may be attributed to the reduction of the reflectance on the glass because the presence of the ITO film.

To investigated the effect of directly sputter titanium dioxide as dopant and compare with the previous results where 


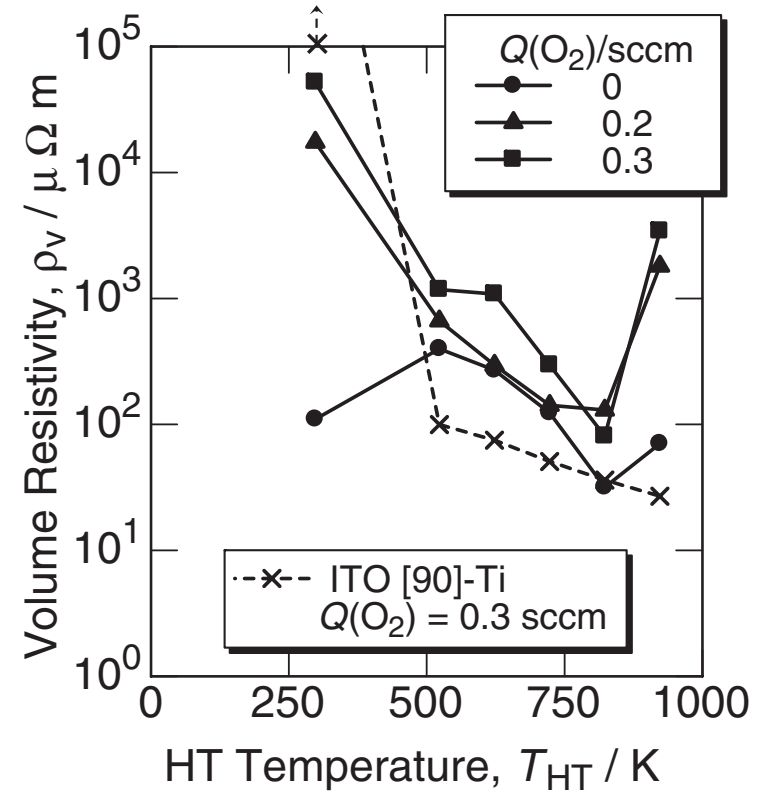

Fig. 9 Effects of $T_{\mathrm{HT}}$ and $Q\left(\mathrm{O}_{2}\right)$ on the $\rho_{\mathrm{V}}$ of $\mathrm{TiO}_{2}$ doped ITO [50] thin films produced by co-sputtering method.

metallic Ti was sputtered, $\mathrm{TiO}_{2}$ doped ITO [50] films were also produced, as it was mentioned before the reason to use an ITO [50] target at this point is exclusively to reduce the content of $\mathrm{In}_{2} \mathrm{O}_{3}$ at around 48 mass\% for the obtained films.

Figure 9 shows the effects of $T_{\mathrm{HT}}$ and $Q\left(\mathrm{O}_{2}\right)$ on the $\rho_{\mathrm{V}}$ measured for the $\mathrm{TiO}_{2}$ doped ITO [50] films, in addition, the best results obtained from the Ti doped ITO [90] films, under the condition $Q(\mathrm{Ar}) / Q\left(\mathrm{O}_{2}\right)=50 / 0.3$, are shown in the same figure for comparing the effect of using metallic and dioxide titanium as dopant during the sputtering.

According to Fig. 9, the lower $\rho_{\mathrm{V}}$ resulted when nonaddition of oxygen was considered during the co-sputtering of $\mathrm{TiO}_{2}$ doped ITO [50] films, even more for the as-depo. films, by the addition of 0.2 and $0.3 \mathrm{sccm}$ of oxygen, $\rho_{\mathrm{V}}$ resulted to be increased in the order of 100 and 500 times, respectively. For the HT films, $\rho_{\mathrm{V}}$ present similar tendencies each other against $T_{\mathrm{HT}}$ decreasing in the range between 523 to $823 \mathrm{~K}$ and then abruptly increasing at $923 \mathrm{~K}$, this tendency may be ascribed to a combined effect because crystallization of films increases with increasing temperature reducing the defect in trap and improving the hall mobility, nevertheless oxygen vacancies became filled under atmosphere pressure at high temperature decreasing the carrier density and then increasing $\rho_{\mathrm{V}},{ }^{12}$ ) the latter effect might have resulted to be stronger at $923 \mathrm{~K}$. In order to corroborate this tendencies further parameters such as hall mobility and carrier density will be measured for the next investigation.

For the $\mathrm{TiO}_{2}$ doped ITO [50] experiments, the minimum value for $\rho_{\mathrm{V}}$ of $31.7 \mu \Omega \mathrm{m}(\sigma=21 \mathrm{mS})$ was obtained at $823 \mathrm{~K}$ without the addition of oxygen. In comparison to the $\mathrm{Ti}$ doped ITO [90] best result, this value was barely higher. It is also important to note that the $\rho_{\mathrm{V}}$ for the asdepo. film was $110 \mu \Omega \mathrm{m}(\sigma=7 \mathrm{mS})$ which is still an interesting value for some proposes considering that films may be directly produced without the necessity to treated them later at high temperature.

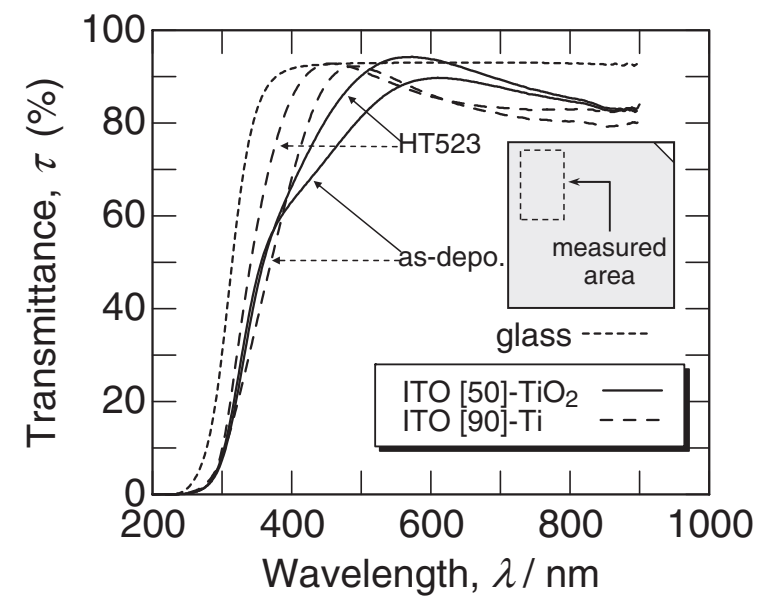

Fig. 10 Effects of $T_{\mathrm{HT}}$ on the $\tau$ of $\mathrm{TiO}_{2}$ doped ITO [50] and Ti doped ITO [90] thin films produced by co-sputtering method, when $Q(\mathrm{Ar}) /$ $Q\left(\mathrm{O}_{2}\right)=50 / 0$ and $50 / 0.3$, respectively.

$\tau$ was measured for the $\mathrm{TiO}_{2}$ doped ITO [50] films produced under the condition of $Q(\mathrm{Ar}) / Q\left(\mathrm{O}_{2}\right)=50 / 0$. Since HT films show an almost identical $\tau$ each other only the results obtained from the as-depo. and HT523 films are shown in Fig. 10. In addition, the as-depo. and HT523 results obtained from Ti doped ITO [90] films under the condition $Q(\mathrm{Ar}) / Q\left(\mathrm{O}_{2}\right)=50 / 0.3$, are shown in the same figure for comparing the effect of using metallic and dioxide titanium as dopant during co-sputtering.

According to Fig. 10, $\mathrm{TiO}_{2}$ doped ITO [50] as-depo. film presents a $\tau$ with a concave form similar to $\mathrm{Ti}$ doped ITO [90] as-depo. film with values between 85 and $90 \%$ although displaced in the $\lambda$ range, between $520 \sim 800 \mathrm{~nm}$, while its corresponding HT523 film shows a much more improved $\tau$ with values over $85 \%$ in the $\lambda$ range between $450 \sim 800 \mathrm{~nm}$ and reaching even values around 93\% at $550 \mathrm{~nm}$ because the presence of $\mathrm{TiO}_{2}$ reduced the reflectance on the glass.

Regarding to crystallization, Fig. 11 shows the results measured on the upper-left part of the $\mathrm{Ti}$ and $\mathrm{TiO}_{2}$ doped ITO films obtained by co-sputtering method having the calculated patterns of $\mathrm{TiO}_{2}, \alpha$-Ti and $\mathrm{In}_{2} \mathrm{O}_{3}$ as standard. According to Fig. 11, some of the representative five peaks assigned to the cubic bixbyte structure of $\operatorname{In}_{2} \mathrm{O}_{3}{ }^{11)}$ start to appear in the HT523 and HT823 samples for Ti and $\mathrm{TiO}_{2}$ doped ITO, respectively, this difference in crystallinity may be ascribed to the concentration of dopants, tin and titanium or oxygen vacancy, trapped at crystalline defects which are related to the composition of the films.

Composition of the co-sputtered $\mathrm{Ti}$ and $\mathrm{TiO}_{2}$ doped ITO films were calculated based on the deposition rate of each target, even when the films present a quite similar mass $\%$ of $\mathrm{In}_{2} \mathrm{O}_{3}$ of 49 and 48 , their mass $\%$ of $\mathrm{SnO}_{2}$ and $\mathrm{TiO}_{2}$, as oxide dopants, are totally different of about 5 and 46, and around 47 and 5 in the $\mathrm{Ti}$ and $\mathrm{TiO}_{2}$ doped ITO films, respectively. According to these results and since even for the films contained 47 mass $\%$ of $\mathrm{SnO}_{2}$ no related peaks were observed, it may be suggested that the presence of large amounts of $\mathrm{TiO}_{2}$ as dopant would improve the crystallinity of bixbite structure. 

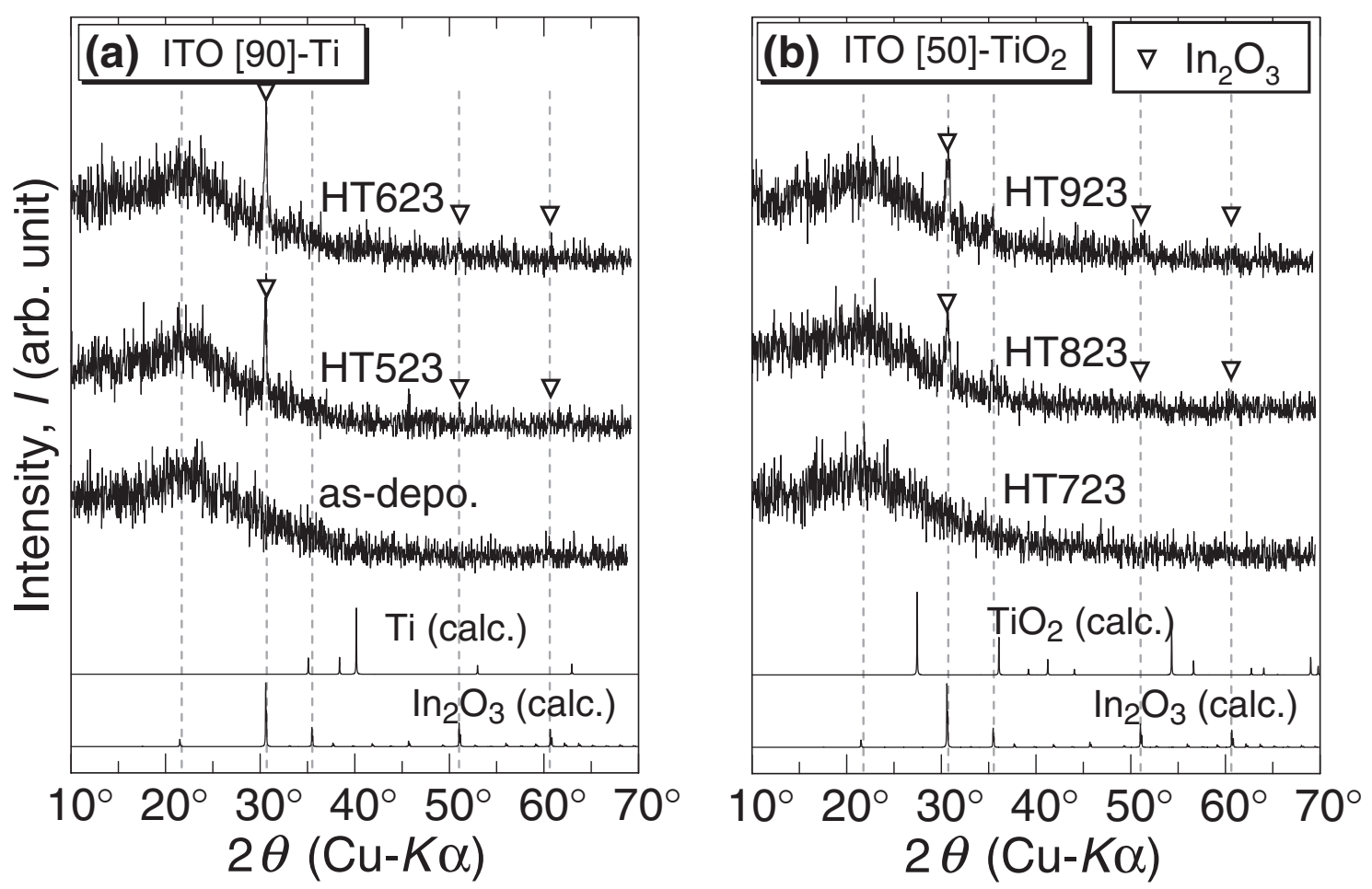

Fig. 11 XRD results for (a) Ti doped ITO [90] and (b) $\mathrm{TiO}_{2}$ doped ITO [50] thin films produced by co-sputtering method, when $Q$ (Ar)/ $Q\left(\mathrm{O}_{2}\right)=50 / 0.3$ and $50 / 0$, respectively.

Finally, surface analyses were taken for the as-depo. and HT lowest $\rho_{\mathrm{V}}$ thin films by using SPM under the DFM and compared each other on the base of root mean square roughness, (RMS) and extended grain diameter, $\left(d_{\mathrm{g}}\right)$. According to the DFM results shown in Fig. 12, RMS, and $d_{\mathrm{g}}$ resulted to be approximately $0.7,1.2$ and $3.6 \mathrm{~nm}$ and 14, 21 and $33 \mathrm{~nm}$ for the Ti doped ITO [90], $\mathrm{TiO}_{2}$ doped ITO [50] and ITO [90] as-depo. films, respectively, and for their lowest temperature crystallized HT523, HT823 and HT523 films, both RMS and $d_{\mathrm{g}}$ increased to 1.0, 1.3 and $6.5 \mathrm{~nm}$ and to 36,41 and $187 \mathrm{~nm}$, respectively. In comparison to as-depo. films, HT ones presented a larger roughness and grain size, and even when those parameters are quite similar for the $\mathrm{HT} \mathrm{Ti}$ and $\mathrm{TiO}_{2}$ doped ITO films. There is an important difference of $300 \mathrm{~K}$ for their lowest temperature crystallization, that different may be decisive at the moment to evaluate the cost of production for the transparent conductive films because lower $T_{\mathrm{HT}}$ means lower production cost.

\section{Summary}

In this study, ITO thin films doped with $\mathrm{Ti}$, as metal and dioxide, were produced by combinatorial and co-sputtering methods. Having fixed the thickness and the content of $\mathrm{In}_{2} \mathrm{O}_{3}$ at about $150 \mathrm{~nm}$ and 48 mass $\%$, respectively, effects of the oxygen flow and heat treatment temperature on the optoelectronical properties of the produced thin films were investigated. The results are summarized as follows:

(1) Combinatorial method resulted to be extremely useful because a multi-evaluation can be carried out on the produced film with a gradient composition on it.
(2) For the Ti and $\mathrm{TiO}_{2}$ doped ITO films produced by cosputtering method, minimum volume resistivities resulted at $Q\left(\mathrm{O}_{2}\right)=0.3$ and 0 , respectively. Oxygen presented a stronger chemical affinity for titanium than that for indium, thus the direct doping of $\mathrm{TiO}_{2}$ resulted easier to control.

(3) For all the experiments, $\rho_{\mathrm{V}}$ decreases with increasing $T_{\mathrm{HT}}$ due to the improvement of $\mathrm{In}_{2} \mathrm{O}_{3}$ bixbyte crystallinity. The best obtained values of $\rho_{\mathrm{V}}=26.9$ and $31.7 \mu \Omega \mathrm{m}$ were obtained at 923 and $823 \mathrm{~K}$ for the $\mathrm{Ti}$ and $\mathrm{TiO}_{2}$ doped ITO film, respectively. In comparison to the ITO [90] HT523, those values are about twice but still promising.

(4) Since titanium dioxide has a higher transmittance in comparison to tin dioxide, $\mathrm{Ti}$ and $\mathrm{TiO}_{2}$ doped ITO films presented an important improvement in their $\tau$, in contrast to ITO [90] films, even as-depo. ones satisfied the required condition of $\tau>85 \%$ in the visible region of the spectrum.

(5) By using titanium as dopant during the production of ITO films the use of $\mathrm{In}_{2} \mathrm{O}_{3}$ might be considerably reduced, nevertheless since heat treatment at high temperature is required an alternative method such as pre heated sputtering (PHS) must be investigated.

\section{Acknowledgments}

The present research was supported by Rare Metal Substitute Materials Development Project in 2007, "Development of technology for reducing indium usage in a transparent conducting electrode" from Ministry of Economy, Trade and Industry (METI) and Rare Metal Substitute 


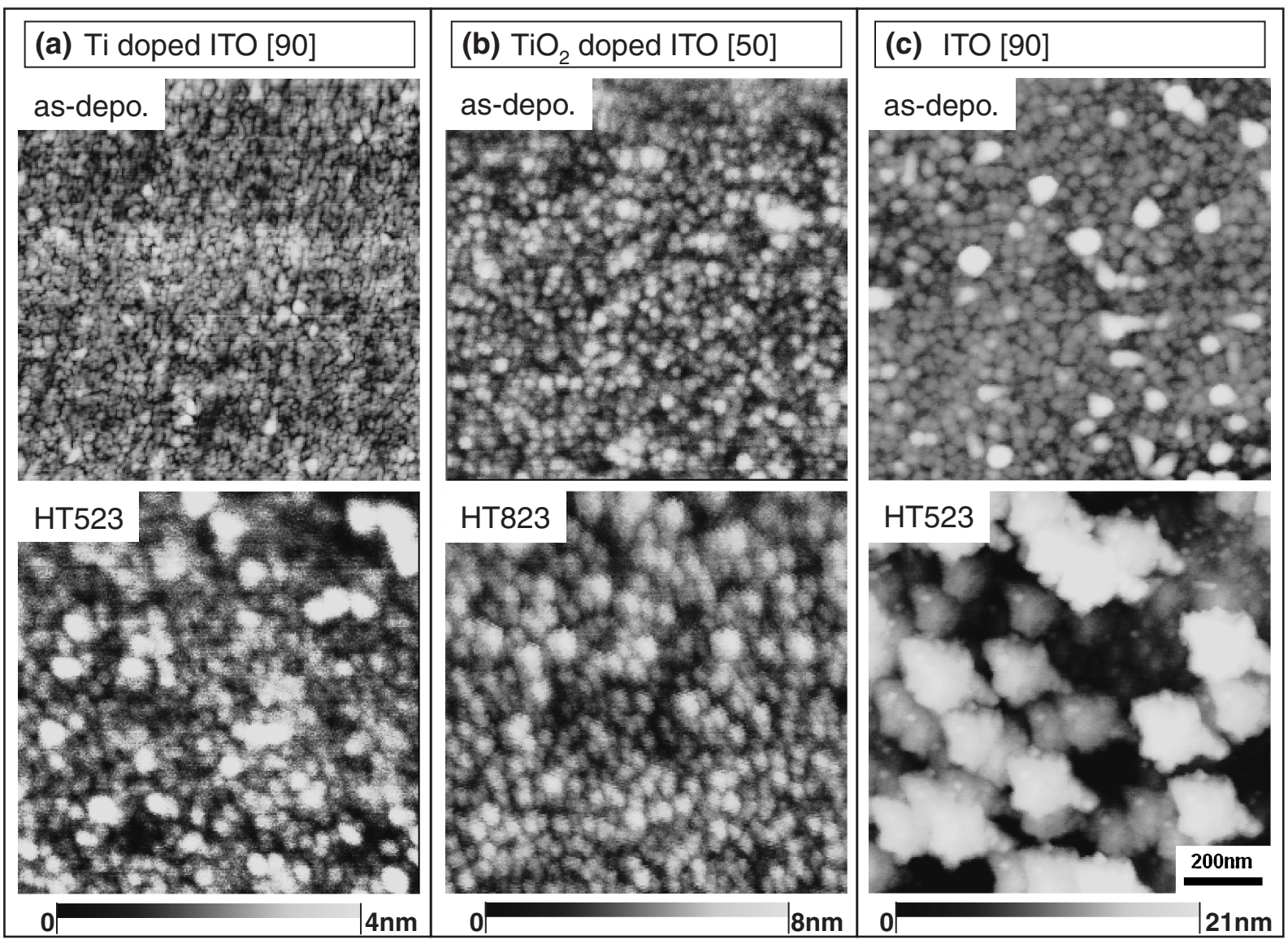

Fig. 12 DFM surface analyses for (a) Ti doped ITO [90], (b) $\mathrm{TiO}_{2}$ doped ITO [50] and (c) ITO [90] as-depo. and HT thin films produced by co-sputtering method, when $Q(\mathrm{Ar}) / Q\left(\mathrm{O}_{2}\right)=50 / 0.3,50 / 0$ and 50/0.2, respectively.

Materials Development Project in 2008, 2009, "Development of technology for reducing indium usage in a transparent conducting electrode" form New Energy and Industrial Technology Development Organization (NEDO), Japan.

\section{REFERENCES}

1) M. Monkade, Z. Fuliang, P. Martinot-Lagarde and G. Durand: Appl. Phys. Lett. 50 (1987) 856-858.

2) C. W. Tang: Appl. Phys. Lett. 48 (1986) 183-185.

3) C. W. Tang and S. A. VanSlyke: Appl. Phys. Lett. 51 (1987) 913-915.

4) I. Hamberg and C. G. Granqvist: J. Appl. Phys. 60 (1986) 123-159.
5) C. Mikolajczak: Proc. 22nd EU PV Conference, (Milan, Italy, 2009).

6) Web site: http://metalprices.com

7) Mineral Commodities Summary: Indium, Geological Survey, USA, (2007) p. 12.

8) D. Cohen: New Scientist 194 (2007) 38-39.

9) J. Barksdale: The Encyclopedia of the Chemical Elements, ed. by Clifford A. Hampel, (Reinhold Book Corporation, New York, 1968) pp. 732-738.

10) L. Voisin, M. Ohtsuka and T. Nakamura: Proc. ICCE-17 International Conference, (Hawaii, USA, 2009).

11) Y. Shigesato, S. Takaki and T. Haranou: Appl. Surf. Sci. 48/49 (1991) 269-275.

12) H. Kim, C. M. Gilmore, A. Piqué, J. S. Horwitz, H. Mattoussi, H. Murata, Z. H. Kafafi and D. B. Chrisey: J. Appl. Phys. 86 (1999) 64516461. 